\title{
Editorial
}

\section{New Trends on Fractional and Functional Differential Equations}

\author{
Dumitru Baleanu, ${ }^{1}$ Ali H. Bhrawy, ${ }^{2}$ and Robert A. Van Gorder ${ }^{3}$ \\ ${ }^{1}$ Department of Mathematics and Computer Sciences, Cankaya University, Yenimahalle, 06810 Ankara, Turkey \\ ${ }^{2}$ Department of Mathematics, Faculty of Science, King Abdulaziz University, Jeddah 21589, Saudi Arabia \\ ${ }^{3}$ Department of Mathematics, University of Central Florida, Orlando, FL 32816, USA
}

Correspondence should be addressed to Dumitru Baleanu; dumitru@cankaya.edu.tr

Received 7 October 2014; Accepted 7 October 2014

Copyright (C) 2015 Dumitru Baleanu et al. This is an open access article distributed under the Creative Commons Attribution License, which permits unrestricted use, distribution, and reproduction in any medium, provided the original work is properly cited.

The fractional and functional differential equations are two hot topics in mathematics, and they have multiple applications in various branches of science and engineering. The combination of these two types of differential equations leads to some interesting results from both theoretical and applied viewpoints.

The purpose of this special issue was to discuss some new algorithms and methods devoted to investigate the fractional and functional differential equations. Below we give a brief survey of the content of this special issue.

Existence theory of positive solutions for second-order singular differential equations with deviating arguments and its parameter dependence were reported. A parallel fast solution for Riesz space fractional reaction-diffusion equation was investigated. Positive solutions for a class of state dependent boundary value problems with fractional order differential operators were found. Step size restrictions for nonlinear stability properties of neutral delay differential equations and Green's function and positive solutions for a second-order singular boundary value problem with integral boundary conditions and a delayed argument were investigated. The dynamics and synchronization of a fractional-order system with complex variables, the existence of solutions for Riemann-Liouville fractional boundary value problem, and the existence of almost periodic solutions for impulsive neutral functional differential equations were other topics of our special issue. Fractional differential equations with fractional impulsive and nonseparated boundary conditions and global well-posedness and longtime decay of fractional NavierStokes equations in Fourier-Besov spaces were reported. Parameter estimation for stochastic differential equations driven by mixed fractional Brownian motion, solutions of a nonlinear Erdelyi-Kober integral equation, and a novel kernel for RBF based neural networks are other subjects of the special issue. Numerical algorithm for the third-order partial differential equation with three-point boundary value problem, modelling the nonlinear wave motion within the scope of the fractional calculus, generalized Kudryashov method for time-fractional differential equations were presented. A novel approach for dealing with partial differential equations with mixed derivatives, the variational iteration transform method for fractional differential equations with local fractional derivative, an efficient collocation method for a class of boundary value problems arising in mathematical physics and geometry, and Cauchy problems for evolutionary pseudo differential equations over p-adic field were analyzed. New ultra spherical wavelets spectral solutions for fractional Riccati differential equations, nonlinear Gronwall-BellmanGamidov integral inequalities and their weakly singular analogues with applications, a new Legendre collocation method for solving atwo-dimensional fractional diffusion equation, computation of spectral parameter of discontinuous Dirac systems with a Gaussian multiplier, and an analytical study of fractional-order multiple chaotic FitzHughNagumo neurons model using multistep generalized differential transform method were interesting reported topics. The small time asymptotics of SPDEs with reflection, the convergence of variational iteration method for solving singular partial differential equations with fractional order, the fourth-order compact difference scheme for the RiemannLiouville and Riesz derivatives, the existence of solutions for fractional boundary value problem with nonlinear derivative dependence, and regularized fractional power parameters for image denoising based on convex solution of fractional heat 
equation were distinct topics of our special issue. Solving the Fokker-Planck equations on Cantor sets using local fractional decomposition method was also reported and an efficient series solution for fractional differential equations was analyzed. Two hybrid methods for solving two-dimensional linear time-fractional partial differential equations and the application of local fractional series expansion method to solve Klein-Gordon equations on Cantor sets are subjects investigated by the authors of our special issue. A pseudospectral algorithm for solving multipantograph delay systems on a semi-infinite interval using Legendre rational functions was developed and a modified groundwater flow model using the space time Riemann-Liouville fractional derivatives approximation was reported.

Dumitru Baleanu

Ali H. Bhrawy

Robert A. Van Gorder 


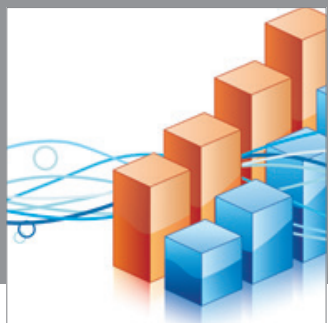

Advances in

Operations Research

mansans

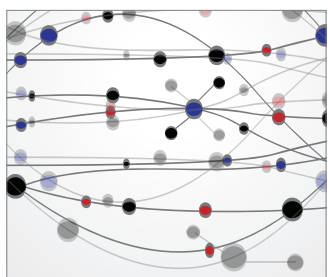

The Scientific World Journal
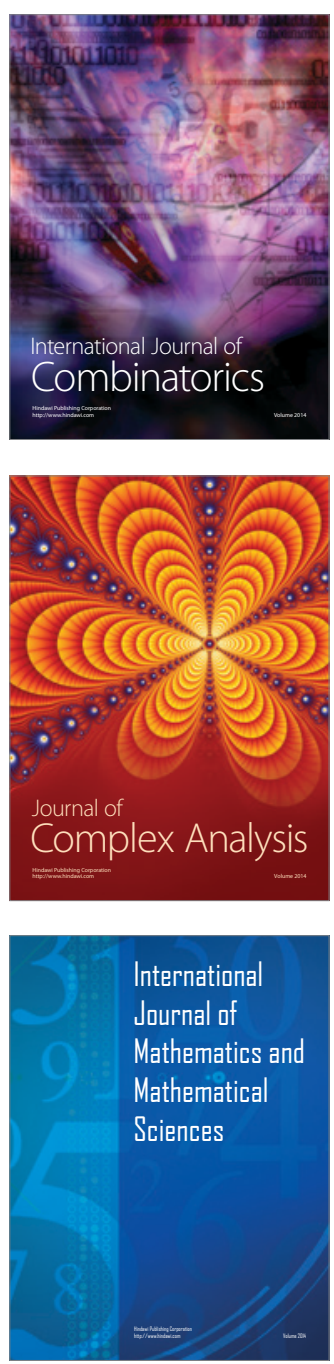
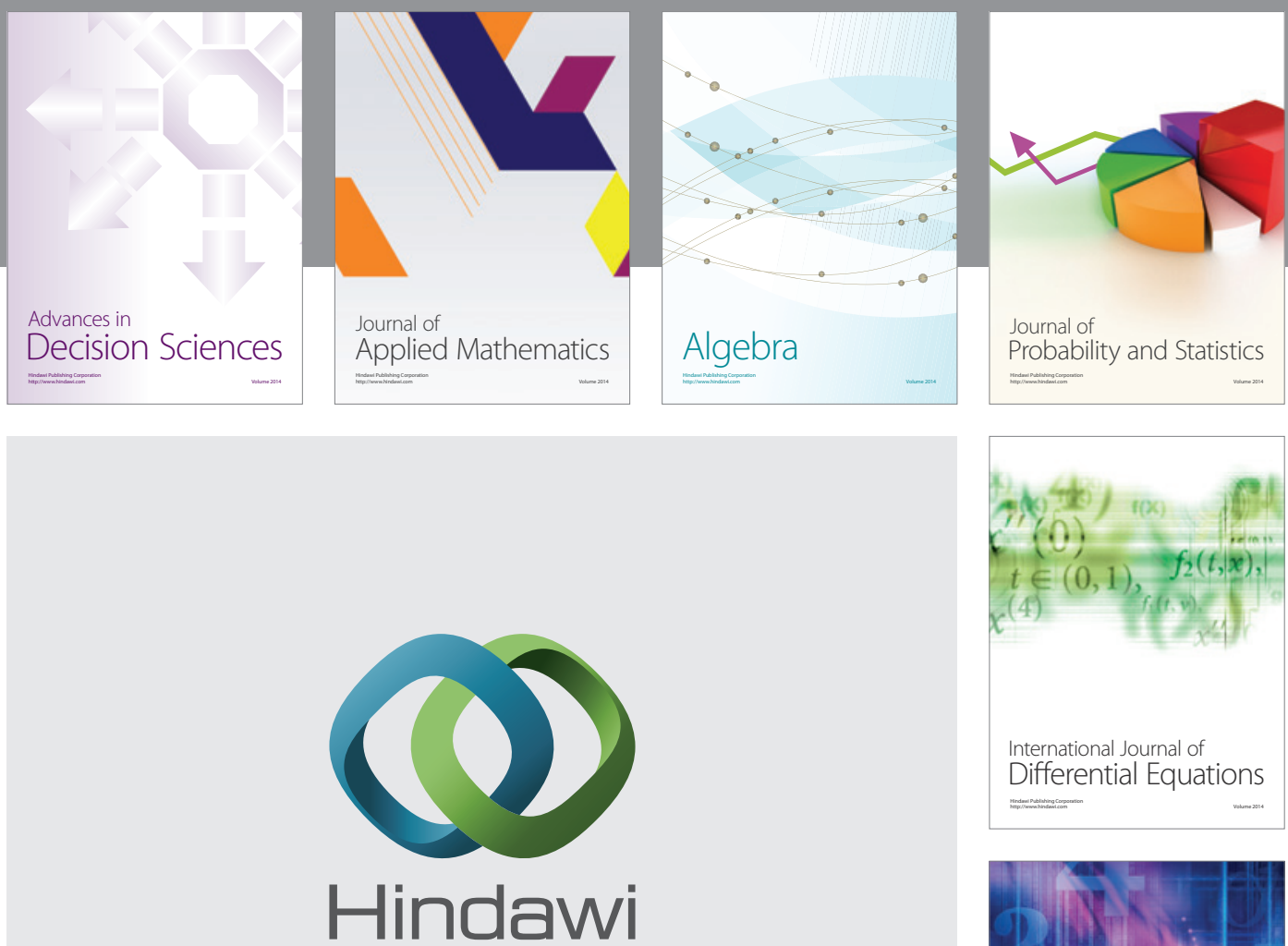

Submit your manuscripts at http://www.hindawi.com
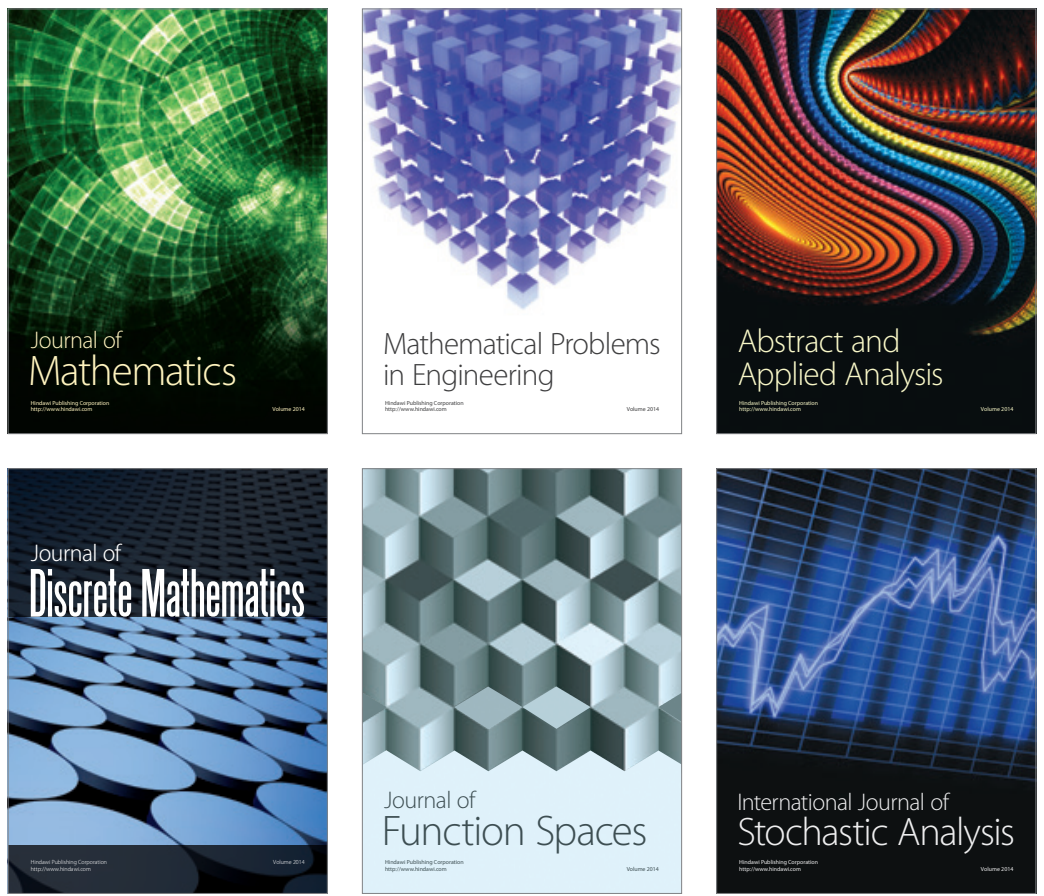

Journal of

Function Spaces

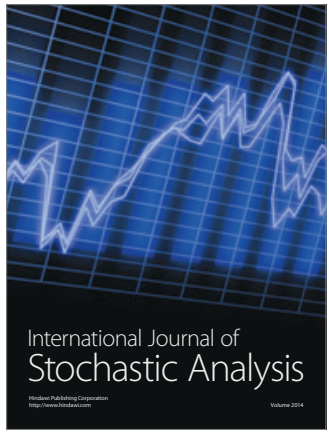

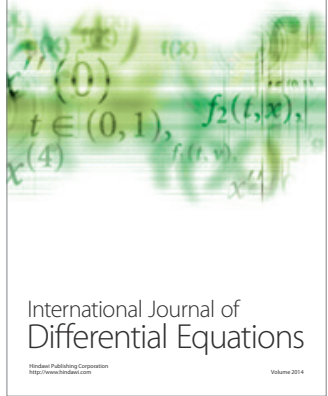
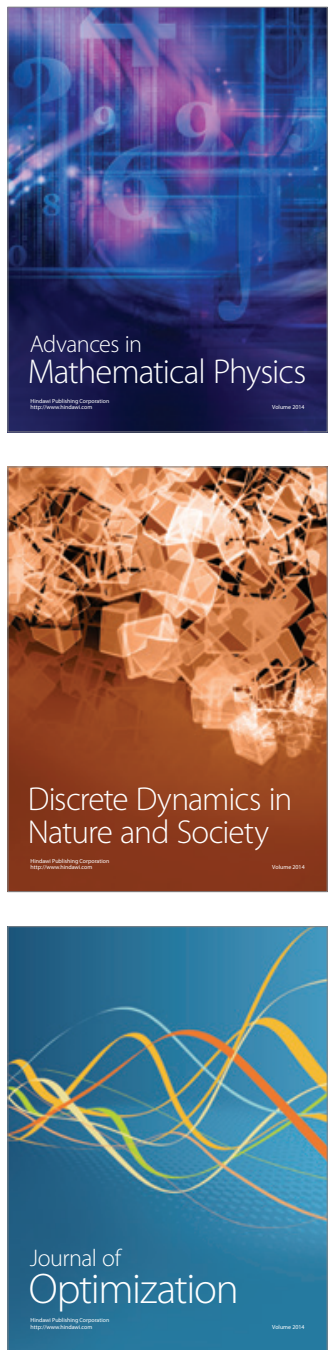